\title{
MS33-05 | Exploring the Dynamic Gas Adsorption Behaviour of a Family of Coordination Polymers through in situ Diffraction Techniques
}

Roseveare, Thomas (The University of Sheffield, Sheffield, GBR); Warren, Mark (Diamond Light Source, Oxfordshire, GBR); Thompson, Stephen (Diamond Light Source, Oxfordshire, GBR); Brammer, Lee (The University of Sheffield, Sheffield, GBR)

Coordination polymers consist of inorganic coordination nodes connected by organic linkers. The construction of these frameworks leads to crystalline materials with void space present for the inclusion of gaseous and solutionphase guests. Typically these materials are considered to be rigid networks, but a growing number of materials have demonstrated dynamic behaviour in response to external stimuli. Typically, dynamic behaviour is inferred by complementary techniques. For example, isothermal gas adsorption studies often result in stepped isotherms or hysteresis suggesting a dynamic structural change. Without in situ structural studies, however, it is not possible to definitively show that a stepped adsorption isotherm is due to dynamic structural behaviour of the material. The development of gas cells has made these in situ diffraction experiments more accessible, with control of the pressure dosing allowing for recording structural data over a full pressure range.

The work presented focuses on a family of one-dimensional coordination polymers $\left(\left[\mathrm{Cu}_{2}(4-\mathrm{Xbz})_{4}(\mathrm{pyz})\right]_{\mathrm{n}}\right.$, where $\mathrm{bz}=$ benzoate, $\mathrm{X}=\mathrm{F}, \mathrm{Cl}, \mathrm{Br}$ or I and pyz=pyrazine). Prior work on this family of coordination polymers demonstrated, by volumetric gas adsorption, that they display guest inclusion properties dependent on the halogen present on the benzoate ligand. This work, therefore, sought to better understand how these polymers changed as a function of increasing gas pressure by conducting in situ single-crystal and powder synchrotron diffraction studies at Diamond Light Source. This work also explored how functionalising the pyrazine linker affects the uptake properties by using both volumetric gas adsorption and in situ diffraction techniques. 